\title{
Determination of the Leptonic Branching Ratios of the $\mathrm{Z}$
}

3 November 1989

\section{The ALEPH Collaboration}

D. Decamp, B. Deschizeaux, J.-P. Lees, M.-N. Minard

Laboratoire de Physique des Particules (LAPP), IN ${ }^{2} P^{3}$-CNRS, 74019 Annecy-le-Vieux Cedex, France

J.M. Crespo, M. Delfino, E. Fernandez ${ }^{(1)}$, M. Martinez, R. Miquel, Ll.M. Mir, S. Orteu, A. Pacheco, J.A. Perlas, E. Tubau

Laboratorio de Fisica de Altas Energias, Universidad Autonoma de Barcelona, 08193 Bellaterra (Barcelona), Spain ${ }^{(10)}$

M.G. Catanesi, M. de Palma, A. Farilla, G. Iaselli, G. Maggi, A. Mastrogiacomo, S. Natali, S. Nuzzo, A. Ranieri,

G. Raso, F. Romano, F. Ruggieri, G. Selvaggi, L. Silvestris, P. Tempesta, G. Zito

INFN Sezione di Bari e Dipartimento di Fisica dell' Università, 70126 Bari, Italy

Y. Chen, H. Hu, D. Huang, J. Lin, T. Ruan, T. Wang, W. Wu, Y. Xie, D. Xu, R. Xu, J. Zhang, W. Zhao

Institute of High-Energy Physics, Academia Sinica, Beijing, The People's Republic of China(11)

H. Albrecht ${ }^{(2)}$, F. Bird, E. Blucher, T. Charity, H. Drevermann, Ll. Garrido, C. Grab, R. Hagelberg, S. Haywood,

B. Jost, M. Kasemann, G. Kellner, J. Knobloch, A. Lacourt, I. Lehraus, T. Lohse, D. Lüke (2), A. Marchioro,

P. Mato, J. May, V. Mertens, A. Minten, A. Miotto, P. Palazzi, M. Pepe-Altarelli, F. Ranjard, J. Richstein ${ }^{(3)}$,

A. Roth, J. Rothberg ${ }^{(4)}$, H. Rotscheidt, W. von Rüden, R. St.Denis, D. Schlatter, M. Takashima, M. Talby,

H. Taureg, W. Tejessy, H. Wachsmuth, S. Wheeler, W. Wiedenmann, W. Witzeling, J. Wotschack European Laboratory for Particle Physics (CERN), 1211 Geneva 23, Switzerland.

Z. Ajaltouni, M. Bardadin-Otwinowska, A. Falvard, P. Gay, P. Henrard, J. Jousset, B. Michel, J-C. Montret, D. Pallin, P. Perret, J. Prat, J. Proriol, F. Prulhière

Laboratoire de Physique Corpusculaire, Université Blaise Pascal, IN ${ }^{2} P^{3}$-CNRS, Clermont-Ferrand, 63177 Aubière, France

H. Bertelsen, F. Hansen, J.D. Hansen, J.R. Hansen, P.H. Hansen, A. Lindahl, B. Madsen, R. Møllerud, B.S. Nilsson,

G. Petersen

Niels Bohr Institute, 2100 Copenhagen, Danmark ${ }^{(12)}$

E. Simopoulou, A. Vayaki

Nuclear Research Center Demokritos (NRCD), Athens, Greece

J. Badier, D. Bernard, A. Blondel, G. Bonneaud, J. Bourotte, F. Braems, J.C. Brient, M.A. Ciocci, R. Guirlet,

P. Miné, A. Rougé, H. Videau, I. Videau ${ }^{(1)}$, D. Zwierski Laboratoire de Physique Nucléaire et des Hautes Energies, Ecole Polytechnique, IN ${ }^{2} P^{3}-C N R S, 91128$ Palaiseau Cedex, France

D.J. Candlin

Department of Physics, University of Edinburgh, Edinburgh EH9 3JZ, United Kingdom(13) 
A. Conti, G. Parrini

Dipartimento di Fisica, Università di Firenze, INFN Sezione di Firenze, 50125 Firenze, Italy

M. Corden, C. Georgiopoulos, J.H. Goldman, M. Ikeda, J. Lannutti, D. Levinthal ${ }^{(18)}$, M. Mermikides, L. Sawyer Supercomputer Computations Research Institute and Dept. of Physics, Florida State University, Tallahassee FL 32306, USA(15)(16)(17)

A. Antonelli, R. Baldini, G. Bencivenni, G. Bologna ${ }^{(5)}$, F. Bossi, P. Campana, G. Capon, V. Chiarella, G. De Ninno, B. D'Ettorre-Piazzoli ${ }^{(6)}$, G. Felici, P. Laurelli, G. Mannocchi ${ }^{(6)}$, F. Murtas, G.P. Murtas, G. Nicoletti, P. Picchi ${ }^{(5)}$, P. Zografou Laboratori Nazionali dell'INFN (LNF-INFN), 00044 Frascati, Italy

B. Altoon, O. Boyle, A.J. Flavell, A.W. Halley, I. ten Have, J.L. Hearns, I.S. Hughes, J.G. Lynch, D.J. Martin, R. O'Neill, C. Raine, J.M. Scarr, K. Smith(1), A.S. Thompson Department of Physics and Astronomy, University of Glasgow, Glasgow G12 8QQ, United Kingdom ${ }^{(13)}$

B. Brandl, O. Braun, R. Geiges, C. Geweniger ${ }^{(1)}$, P. Hanke, V. Hepp, E.E. Kluge, Y. Maumary, M. Panter, A. Putzer, E. Rensch, A. Stahl, K. Tittel, M. Wunsch Institut für Hochenergiephysik, Universität Heidelberg, 6900 Heidelberg, Fed. Rep. of Germany ${ }^{(19)}$

G.J. Barber, A.T. Belk, R. Beuselinck, D.M. Binnie, W. Cameron (1), M. Cattaneo, P.J. Dornan, S. Dugeay, R.W. Forty, D.N. Gentry, J.F. Hassard, D.G. Miller, D.R. Price, J.K. Sedgbeer, G. Taylor, I.R. Tomalin Department of Physics, Imperial College, London SW7 2BZ, United Kingdom ${ }^{(13)}$

P. Girtler, D. Kuhn, G. Rudolph Institut für Experimentalphysik, Universität Innsbruck, 6020 Innşbruck, Austria (21)

C.K. Bowdery (1), T.J. Brodbeck, A.J. Finch, F. Foster, G. Hughes, N.R. Keemer, M. Nuttall, B.S. Rowlingson, T. Sloan, S.W. Snow Department of Physics, University of Lancaster, Lancaster LA1 $4 Y B$, United Kingdom ${ }^{(13)}$

T. Barczewski, L.A.T. Bauerdick, K. Kleinknecht ${ }^{(1)}$, B. Renk, S. Roehn, H.-G. Sander, M. Schmelling, F. Steeg Institut für Physik, Universität Mainz, 6500 Mainz, Fed. Rep. of Germany (19)

J-P. Albanese, J-J. Aubert, C. Benchouk, A. Bonissent, F. Etienne, R. Nacasch, P. Payre, B. Pietrzyk ${ }^{(1)}$, Z. Qian Centre de Physique des Particules, Faculté des Sciences de Luminy, IN ${ }^{2} P^{3}$-CNRS, 13288 Marseille, France

W. Blum, P. Cattaneo, M. Comin, G. Cowan, B. Dehning, H. Dietl, M. Fernandez-Bosman, D. Hauff, A. Jahn, E. Lange, G. Lütjens, G. Lutz, W. Männer, H-G. Moser, Y. Pan, R. Richter, A.S. Schwarz, R. Settles, U. Stiegler, U. Stierlin, G. Stimpfl(7), J. Thomas, G. Waltermann

Max-Planck-Institut für Physik und Astrophysik, Werner-Heisenberg-Institut für Physik, 8000 München, Fed. Rep. of Germany ${ }^{(19)}$

G. de Bouard, J. Boucrot, O. Callot, A. Cordier, M. Davier, G. Ganis, J.-F. Grivaz, Ph. Heusse, P. Janot, V. Journé, D.W. Kim, J. Lefrançois, D. Lloyd-Owen, A.-M. Lutz, P. Marotte, J.-J. Veillet, F. Zomer Laboratoire de l'Accéléateur Linéaire, Université de Paris-Sud, IN P $^{3}$-CNRS, 91405 Orsay Cedex, France

S.R. Amendolia, G. Bagliesi, G. Batignani, L. Bosisio, U. Bottigli, C. Bradaschia, I. Ferrante, F. Fidecaro, L. Foà(1), E. Focardi, F. Forti, A. Giassi, M.A. Giorgi, F. Ligabue, A. Lusiani, E.B. Mannelli, P.S. Marrocchesi, A. Messineo, F. Palla, G. Sanguinetti, S. Scapellato, J. Steinberger, R. Tenchini, G. Tonelli, G. Triggiani 
Dipartimento di Fisica dell'Università, INFN Sezione di Pisa, e Scuola Normale Superiore,56010 Pisa, Italy

J.M. Carter, B.J. Green, M.G. Green, A.K. McKemey, P.V. March, T. Medcalf, M.R. Saich, J.A. Strong(1), R.M. Thomas, T. Wildish Department of Physics, Royal Holloway \& Bedford New College, University of London, Surrey TW20 OEX, United Kingdom ${ }^{(13)}$

D.R. Botterill, R.W. Clifft, T.R. Edgecock, M. Edwards, S.M. Fisher, J. Harvey, D.L. Hill, T.J. Jones, M. Morrissey, P.R. Norton, D.P. Salmon, J.C. Thompson High-Energy Physics Division, Rutherford Appleton Laboratory, Chilton, Didcot, OXON OX11 OQX, United Kingdom ${ }^{(13)}$

B. Bloch-Devaux, P. Colas, C. Klopfenstein, E. Lançon, E. Locci, S. Loucatos, L. Mirabito, E. Monnier, P. Perez, F. Perrier, J. Rander, J.-F. Renardy, A. Roussarie, J.-P. Schuller Département de Physique des Particules Élémentaires, CEN-Saclay, 91191 Gif-sur-Yvette Cedex, France ${ }^{(20)}$

J.G. Ashman, C.N. Booth, F. Combley, M. Dinsdale, J. Martin, D. Parker, L.F. Thompson Department of Physics, University of Sheffield, Sheffield S3 7RH, United Kingdom(13)

S. Brandt, H. Burkhardt, C. Grupen, H. Meinhard, E. Neugebauer, U. Schäfer, H. Seywerd, K. Stupperich Fachbereich Physik, Universität Siegen, 5900 Siegen, Fed. Rep. of Germany(19)

B. Gobbo, F. Liello, E. Milotti, F. Ragusa ${ }^{(8)}$, L. Rolandi ${ }^{(1)}$

Dipartimento di Fisica, Università di Trieste e INFN Sezione di Trieste, 34127 Trieste, Italy

L. Bellantoni, J.F. Boudreau, D. Cinabro, J.S. Conway, D.F. Cowen, Z. Feng, J.L. Harton, J. Hilgart, R.C. Jared ${ }^{(9)}$, R.P. Johnson, B.W. LeClaire, Y.B. Pan, T. Parker, J.R. Pater, Y. Saadi, V. Sharma, J.A. Wear, F.V. Weber, Sau Lan Wu, S.T. Xue, G. Zobernig

Department of Physics, University of Wisconsin, Madison, WI 53706, USA (14)

(Submitted to Physics Letters.)

\footnotetext{
${ }^{1}$ Now at CERN.

${ }^{2}$ Permanent address: DESY, Hamburg, Fed. Rep. of Germany.

${ }^{3}$ Now at Lecroy, Geneva.

${ }^{4}$ On leave of absence from University of Washington, Seattle, WA 98195.

${ }^{5}$ Also Istituto di Fisica Generale, Università di Torino, Torino, Italy.

${ }^{6}$ Also Istituto di Cosmo-Geofisica del C.N.R., Torino, Italy.

${ }^{7}$ Now at FSU.

${ }^{8}$ Now at INFN Milano.

${ }^{\circ}$ Permanent address: LBL, California, USA.

${ }^{10}$ Supported by CAICYT, Spain.

"Supported by the National Science Foundation of China.

${ }^{12}$ Supported by the Danish Natural Science Research Council.

${ }^{13}$ Supported by the UK Science and Engineering Research Council.

${ }^{14}$ Supported by the US Department of Energy, contract DE-AC02-76ER00881.

${ }^{16}$ Supported by the US Department of Energy, contract DE-FG05-87ER40319.

${ }^{16}$ Supported by the NSF, contract PHY-8451274.

${ }^{17}$ Supported by the US Department of Energy, contract DE-FC0S-85ER250000.

${ }^{18}$ Supported by SLOAN fellowship, contract BR 2703.

${ }^{10}$ Supported by the Bundesministerium für Forschung und Technologie, Fed. Rep. of Germany.

${ }^{20}$ Supported by the Institut de Recherche Fondamentale du C.E.A..

${ }^{21}$ Supported by Fonds zur Förderung der wissenschaftlichen Forschung, Austria.
} 


\begin{abstract}
The ratios of the numbers of $Z$ bosons decaying to $\mathrm{e}^{+} \mathrm{e}^{-}, \mu^{+} \mu-$ and $\tau+\tau-$ pairs to the number decaying to hadrons have been measured. The branching ratios and partial widths for each channel were determined and found to be equal, consistent with lepton universality. The mean leptonic branching ratio was found to be $0.0321 \pm 0.0013$ and the leptonic partial width to be $85.4 \pm 5.3 \mathrm{MeV}$. The partial widths for hadronic decays and for invisible decays were deduced to be $1833 \pm 116 \mathrm{MeV}$ and $569 \pm 92 \mathrm{MeV}$, respectively. The number of light neutrino types, assuming only the standard model value for the ratio $\Gamma_{e} / \Gamma_{v}$, was found to be $3.35 \pm 0.41$.
\end{abstract}




\section{Introduction}

The advent of the LEP $\mathrm{e}^{+} \mathrm{e}^{-}$collider [1] allows the decays of the $\mathrm{Z}$, the neutral weak boson [2], to be studied in more detail than ever before. In this paper we present measurements of the ratios of the numbers of $\mathrm{e}^{+} \mathrm{e}^{-}, \mu^{+} \mu^{-}$and $\tau+\tau$ - decays to hadronic decays of the $Z$. The data were taken at centre of mass energies close to the peak of the $\mathrm{Z}$ resonance, for which the line shape has been measured previously in this experiment [3]. These ratios, together with the peak cross section to hadrons, allow the leptonic, hadronic and invisible branching ratios to be determined. The partial widths of the $\mathrm{Z}$ are then obtained using the measured total width.

The experiment was performed at the LEP $\mathrm{e}^{+} \mathrm{e}^{-}$collider at CERN using the ALEPH apparatus $[3,4]$ to detect the decays of the $Z$. The data were taken in the first long run of LEP ending in October 1989. For this analysis the centre of mass energy was restricted to the range 90 to $92.5 \mathrm{GeV}$ i.e. close to the $\mathrm{Z}$ peak and the integrated luminosity was $104 \mathrm{nb}^{-1}$. The parts of the ALEPH detector relevant to this analysis are shown in fig. 1 . They consist of an inner tracking chamber (ITC) and a time projection chamber (TPC) inside a magnetic field of $1.5 \mathrm{~T}$. This system served to detect and momentum analyse the charged particle tracks produced by the annihilation of the colliding electrons and positrons. External to the TPC, an electromagnetic calorimeter (ECAL) was used to measure the energy of electrons and to detect and measure photons. The whole of this system is situated inside a superconducting coil. A hadronic calorimeter (HCAL), outside the coil, was used to measure the energy of hadrons and to identify muons.

The apparatus was triggered independently by several separate conditions, three of which were important for the detection of $Z$ decays. The first demanded a total deposition of energy greater than $6 \mathrm{GeV}$ in the ECAL central section (the barrel), or greater than $3 \mathrm{GeV}$ in either ECAL endcap, or greater than $1 \mathrm{GeV}$ in both endcaps. The second trigger required a charged track in the ITC associated in azimuth with energy deposition of more than $1 \mathrm{GeV}$ in the ECAL. The third trigger was defined by a coincidence between any of the HCAL azimuthal segments with a corresponding azimuthal segment of the ITC. A signal in an HCAL segment was registered if hits were recorded at a depth of at least $30 \mathrm{~cm}$ of iron in the barrel region or at least $55 \mathrm{~cm}$ of iron in the endcap region. A signal in any ITC segment was registered by at least five of its eight cylindrical wire layers. For each trigger, flags were set in the data to indicate the trigger source and its azimuthal segment. The trigger efficiency was measured for each decay mode by comparing the different triggers, as discussed below.

The data were processed through a chain of computer programmes in order to perform the operations of pattern recognition, track reconstruction and momentum analysis. Good tracks were each required to have a polar angle $\theta$ with $|\cos \theta|<0.95$, to be reconstructed from 
at least four TPC hits and to originate from within a cylinder, centred on the interaction point, radius $2 \mathrm{~cm}$ and length $20 \mathrm{~cm}$ along the beam. The reconstruction efficiency for isolated tracks was measured by selecting Bhabha events $\left(\mathrm{e}^{+} \mathrm{e}^{-} \rightarrow \mathrm{e}^{+} \mathrm{e}^{-}\right)$defined by ECAL clusters of more than $35 \mathrm{GeV}$ energy. The fraction of these in which both tracks were reconstructed was found to be $99.0 \pm 0.6 \%$, in good agreement with the predicted fraction from a Monte Carlo simulation of $99.2 \pm 0.1 \%$. Thus correction for track reconstruction efficiency was unnecessary since the observed small losses were well simulated by the Monte Carlo.

Hadronic decays of the $\mathrm{Z}$ were selected, as in [3], by requiring a minimum of five charged tracks which carried in total at least $10 \%$ of the centre of mass energy. A total of 2619 $Z$ to hadron decays satisfied these selection criteria. The acceptance for these decays was calculated to be $0.975 \pm 0.006$ [3] from a Monte Carlo simulation in which the generated digitizations were passed through the same reconstruction programmes as the data.

The lepton final states of the $\mathrm{Z}$ are distinguished from the hadronic final states by their low multiplicity. For a first lepton preselection the number of charged tracks was required not to exceed six. This limit was chosen to retain leptonic decays of the $Z$ which were accompanied by $\mathrm{e}^{+} \mathrm{e}^{-}$pairs from photon conversions and to have high efficiency for tau decays. Further procedures were then implemented to identify the individual leptonic decay channels, as described below.

\section{The Decay Mode $\mathrm{Z} \rightarrow \mathrm{e}^{+} \mathrm{e}^{-}$.}

The events for this process were separated from the other reactions by a combination of cuts on charged particle multiplicity, particle momenta and energy deposition in ECAL. Events were retained if there were at least two tracks and the sum of the momenta of the two most energetic tracks exceeded $35 \mathrm{GeV} / \mathrm{c}$. The sum of the ECAL energies associated with these two tracks was required to exceed $40 \mathrm{GeV}$ and their acollinearity to be less than 20 degrees. Furthermore, the scattering angle, $\eta$, defined as the angle between the beam and the bisector of the external angle of the two tracks, was required to satisfy $|\cos \eta|<0.90$. The resulting sample contained 168 events.

With the cuts listed above, the acceptance for events generated with the programme BABAMC [5] and passed through a Monte Carlo simulation and reconstruction was $93.5 \%$. The loss was made up of $2.5 \%$ due to the acollinearity cut, and the remainder from the momentum and energy cuts. The systematic error on this acceptance was estimated to be $2 \%$, mainly due to the uncertainty in the effect of the energy cut. This was obtained by varying the cuts and observing the change in the acceptance. The change in the measured number of events was well reproduced by the Monte Carlo simulation, showing that the acceptance and backgrounds were well understood. 
The angular distribution for these events, corrected bin by bin for the acceptance, is shown in fig. 2. The contribution from $t$ channel photon exchange must be subtracted to obtain the number of $Z \rightarrow \mathrm{e}^{+} \mathrm{e}^{-}$events. Because this contribution peaks in the forward direction, only the region $\cos \eta<0.7$ was used to extract the $Z->\mathrm{e}^{+} \mathrm{e}^{-}$signal. The $t$ channel cross section was evaluated by first generating $\mathrm{e}^{+} \mathrm{e}^{-}$and $\mu^{+} \mu^{-}$events with BABAMC and MUONMC [5]. The Monte Carlo events were generated at the experimental energies to account for the change in the interference effects with energy. At the $Z$ pole these interference effects disappear and they largely cancel when averaging data taken equally above and below the $Z$ peak. The Monte Carlo programmes are first order generators, identical except for the $t$ channel effects which can therefore be extracted to the same first order by comparing the event distributions. The generated events were used only to obtain the shape of the $t$ channel contribution, which was then normalised to reproduce the observed excess of events in the forward direction. The final correction was $15.3 \%$ with interference effects contributing only $1.5 \%$. .

The trigger efficiency was measured by observing the number of times the ITC-ECAL and ECAL energy trigger flags were both set. It was found to be $100 \%$ and no correction was applied. The correction for the $\tau$ background was estimated from a Monte Carlo calculation to be $(4.5 \pm 1.0) \%$. Correcting for this and for the extrapolation into the unmeasured angular intervals $\cos \eta<-0.9$ and $\cos \eta>0.7$ (assuming a $1+\cos ^{2} \eta$ distribution) gives the corrected number of events to be $126.7 \pm 15.7$ (stat) \pm 3.4 (syst). Here the systematic error represents the uncertainties of acceptance $(2 \%), \tau$ subtraction $(1 \%)$ and the $t$ channel subtraction $(1.5 \%)$ added in quadrature. The ratio of the number of electronic to hadronic decays of the $Z$ is then found to be

$$
P_{e}=\frac{\Gamma_{e}}{\Gamma_{h}}=0.0472 \pm 0.0061
$$

Here $\Gamma_{\mathrm{e}}$ is the electronic and $\Gamma_{\mathrm{h}}$ the hadronic decay width of the $\mathrm{Z}$. The systematic and statistical errors have been added in quadrature.

The Decay Mode $\mathbf{Z} \rightarrow \mu^{+} \mu^{-}$.

The events for this process were separated from the preselected lepton sample using only the HCAL barrel data, corresponding to polar angle satisfying $|\cos \theta|<0.61$, since the HCAL endcap digital readout was not yet operational. Cuts were applied to eliminate $\tau^{+} \tau$ decays from the sample by demanding that the particle with the largest momentum had at least $35 \mathrm{GeV}$, that the second largest momentum had at least $22 \mathrm{GeV}$ and that the angle of acollinearity was less than $15^{\circ}$. According to Monte Carlo studies using the KORALZ generator [6], this cut retained ( $97.9 \pm 0.2) \%$ of the $\mu$ pairs, $(1.4 \pm 0.1) \%$ of the $\tau$ pairs 
and the majority of the e pairs. After these cuts the background from two photon interactions and from hadronic $\mathrm{Z}$ decay was negligible.

The $\mu^{+} \mu^{-}$pairs were then separated from the $\mathrm{e}^{+} \mathrm{e}^{-}$pairs by using the muon identification capability of the HCAL barrel. An event was selected as a $\mu^{+} \mu^{-}$pair if at least one of the two tracks was identified as a muon, i.e. it had produced an ITC-HCAL trigger in that segment and had penetrated to a depth of more than $30 \mathrm{~cm}$ of iron in HCAL. We observed 57 such events, 22 with only one and 35 with both muons identified. The overall trigger and identification efficiency for $\mu^{+} \mu^{-}$events in the accepted polar angular region was found to be $0.95 \pm 0.03$.

Figure 3 shows a scatter plot of the distance of closest approach to the beam line (d) versus the corresponding distance along the beam from the interaction point $(z)$, for each muon track in the final sample. It can be seen that all of these muons come from the beam interaction region, whereas cosmic ray muons would populate the entire plot. In figure 4 the $z$ distribution for the tracks of the final sample is compared to that of the preselected sample. The entries in the preselected sample with $|z|>4 \mathrm{~cm}$ are mostly due to the cosmic ray background, which is found to give a negligible contribution to the final sample.

After correcting for the geometrical acceptance (52.3\%) and the $\tau$ background $(0.6 \%)$, the total number of $\mu$ pairs was found to be $116.8 \pm 15.8$ (stat) \pm 3.0 (syst). The systematic error was determined by changing the kinematic cuts. The main contribution came from a systematic inaccuracy in the momentum scale, which is currently being calibrated, of up to $10 \%$ at $45 \mathrm{GeV}$. Thus the ratio of the number of $\mathrm{Z} \rightarrow \mu^{+} \mu^{-}$decays to the number of $\mathrm{Z}$ decays to hadrons is

$$
\mathrm{P}_{\mu}=\frac{\Gamma_{\mu}}{\Gamma_{\mathrm{h}}}=0.0433 \pm 0.0060
$$

The Decay Mode $Z \rightarrow \tau+\tau \cdot$.

The decays of the $\mathrm{Z} \rightarrow \tau+\tau$ - were chosen from the preselected sample by first demanding events with at least one track with momentum of more than $3 \mathrm{GeV}$. The thrust axis was determined and events were retained if they had at least one track in each hemisphere and a total transverse momentum relative to the beam greater than $2.5 \mathrm{GeV}$ in one or both hemispheres. The acollinearity angle between the axes of the jets defined in each hemisphere was then required to be less than $26^{\circ}$. These cuts eliminated most of the background from two photon interactions. The angle between any track in the jet and the jet axis was required to be less than $18.2^{\circ}$. This eliminated the majority of the residual decays of the $Z$ to hadrons. At this stage, the surviving events consisted mainly of the decays of the $Z$ to leptons. 
Events with single charged tracks in each hemisphere were excluded if both track momenta were larger than $35 \mathrm{GeV}$, or $30 \mathrm{GeV}$ if the tracks were identified in the calorimeters as both electrons or both muons. This eliminated most of the decays of the $\mathrm{Z} \rightarrow \mathrm{e}^{+} \mathrm{e}^{-}$and $\mu^{+} \mu^{-}$. To reinforce this, a cut was also made demanding that the energy in the ECAL associated with each track was less than $30 \mathrm{GeV}$. In addition, to eliminate $\mathrm{e}^{+} \mathrm{e}^{*}$ pairs with final state radiated photons, the summed energy in ECAL was required to be less than $70 \mathrm{GeV}$. Furthermore, to eliminate $\mu^{+} \mu^{-}$pairs with a final state radiated photon, events with two muons and an energy cluster in ECAL were kinematically identified and rejected.

The data and Monte Carlo have been compared and found to be consistent. Fig. 5 shows the total energy spectrum of the charged tracks from the data compared to the predictions of the Monte Carlo simulation. The final sample contained 115 events. The backgrounds in this sample were estimated by Monte Carlo calculation to consist of $1.0 \pm 0.6$ events from two photon interactions and a negligible number of events from the decays of the $Z$ to hadrons. The backgrounds from residual $\mathrm{e}^{+} \mathrm{e}^{-}$and $\mu^{+} \mu^{-}$pairs were estimated to be $4 \pm 3$ and $0 \pm 1$ events, respectively. These were measured from the data by observing the number of times an event identified as an $\mathrm{e}^{+} \mathrm{e}^{-}$or $\mu^{+} \mu^{-}$in one hemisphere was misidentified as a $\tau$ in the opposite hemisphere. The cosmic ray muon background was estimated to be $0.5 \pm 0.2$ events by taking the observed number of events at large values of $d$ (the closest distance of approach to the interaction point) and extrapolating, under the peak at small values of $d$ coming from beam interactions. Correcting for all these backgrounds gives the total number of $\tau+\tau-$ events to be $109.5 \pm 10.8$ (stat) \pm 3.2 (syst), where the systematic error comes from adding in quadrature the errors on the number of background events.

The detection efficiency for $\tau+\tau$ - pair production was calculated to be $0.859 \pm .020$ (syst), from a Monte Carlo simulation which included the effects of all the cuts. The trigger efficiency was measured from the data by comparing the number of events in which only one or both azimuthal segments of the trigger along each $\tau$ direction were set. The average efficiency for a single $\tau$ to produce a trigger was found to be $93.4 \%$. The efficiency for either $\tau$ to produce a trigger was then calculated to be $98.3 \pm 0.7 \%$, taking into account a dead region in the ITC for part of the data. The corrected number of $\tau^{+} \tau$ - events becomes $129.7 \pm 12.7$ (stat) \pm 4.9 (syst), giving

$$
\mathrm{P}_{\tau}=\frac{\Gamma_{\tau}}{\Gamma_{\mathrm{h}}}=0.0483 \pm 0.0051
$$




\section{Discussion of the Results}

The three measured values of the ratio $\mathrm{P}_{\lambda}$, for the decay to each lepton type $\lambda$, are shown in column 2 of table 1 . They are equal within the errors, in agreement with lepton universality. The branching ratios $B_{\lambda}=\Gamma_{\lambda} \Gamma_{z}$ can be deduced from the ratios $P_{\lambda}$ as follows. The cross section for hadron production in $\mathrm{e}^{+} \mathrm{e}^{-}$collisions at the energy of the $\mathrm{Z}$ mass, after correction for initial state radiation, is given by

$$
\sigma_{\mathrm{o}}^{\mathrm{h}}=\frac{12 \pi}{\mathrm{M}_{\mathrm{z}}^{2}} \frac{\Gamma_{\mathrm{e}} \Gamma_{\mathrm{h}}}{\Gamma_{\mathrm{z}}^{2}}=\frac{12 \pi}{\mathrm{M}_{\mathrm{z}}^{2}} \mathrm{~B}_{\mathrm{e}} \frac{\mathrm{B}_{\lambda}}{\mathrm{P}_{\lambda}}
$$

This cross section, the mass and width of the $\mathrm{Z}$ have been measured in this experiment [3] to be $39.1 \pm 1.6 \mathrm{nb}, \mathrm{M}_{\mathrm{z}}=91.18 \mathrm{GeV}$ and $\Gamma_{\mathrm{z}}=2.66 \pm 0.16 \mathrm{GeV}$, respectively. Using these with the measured value of $\mathrm{P}_{\mathrm{e}}$, the electron branching ratio, $\mathrm{B}_{\mathrm{e}}$, can be obtained from equation 4 . Then, substituting this value, $B_{\mu}$ and $B_{\tau}$ can be obtained from the measured values of $P_{\mu}$ and $P_{\tau}$. These values of $B_{c}, B_{\mu}$ and $B_{\tau}$ are given in column 3 of table 1 . The partial widths, $\Gamma_{\lambda}$, are then calculated using the measured value of $\Gamma_{z}$ and are shown in column 4 of table 1 . The quoted errors take account of the correlation between the numbers of hadrons used in the determinations of $\mathrm{P}_{\lambda}$ and $\sigma_{\mathrm{h}}^{0}$, and of the correlation between $\sigma_{h}^{0}$ and $\Gamma_{\mathrm{Z}}$.

Since the values of $\mathrm{P}_{\mathrm{e}}, \mathrm{P}_{\mu}$ and $\mathrm{P}_{\tau}$ are equal within errors, compatible with lepton universality, they have been combined giving $\mathrm{P}_{\mathrm{a}}=0.0466 \pm 0.0034$ for an average charged lepton. Using this value in equation 4 , the mean charged leptonic branching ratio is found to be $\mathrm{B}_{\mathrm{a}}=0.0321 \pm 0.0013$. This corresponds to a partial width, $\Gamma_{\mathrm{a}}=85.4 \pm 5.3 \mathrm{MeV}$, in agreement with the value $83.4 \mathrm{MeV}$ predicted by the Standard Electroweak Model [7]. From this value of $\Gamma_{\mathrm{a}}$, the branching ratio for $\mathrm{Z}$ decays to hadrons is deduced using equation 4 to be $\mathrm{B}_{\mathrm{h}}=0.689 \pm 0.030$ and the partial width $\Gamma_{\mathrm{h}}=1833 \pm 116 \mathrm{MeV}$.

Since the branching ratios add to unity, the ratio $\Gamma_{\mathrm{inv}} / \Gamma_{\mathrm{a}}$ is given by

$$
\frac{\Gamma_{\text {inv }}}{\Gamma_{\mathrm{a}}}=\frac{1}{\mathrm{~B}_{\mathrm{a}}}-\frac{1}{\mathrm{P}_{\mathrm{a}}}-3=6.67 \pm 0.81
$$

where $\Gamma_{\text {inv }}$ is the partial width to invisible decays assumed to be due to neutrinos. The correlations mentioned above lead to cancellations in the error which were previously noted by Feldman [8].

Taking the value $\Gamma_{v} / \Gamma_{\mathrm{a}}=1.99$ [7], expected from the Standard Electroweak Model, the number of neutrino types is $3.35 \pm 0.41$. It should be noted that this ratio is the only assumption from the standard model made in this analysis. The result is still valid if additional unexpected hadronic states were to be present in $Z$ decays. Taking $\Gamma_{\mathrm{a}}$ to be the value measured, 
the invisible width is $569 \pm 92 \mathrm{MeV}$. The values of the branching ratios and partial widths, derived above, are summarised in table 1.

In conclusion, the ratios of the leptonic to hadronic $Z$ decays have been measured for electrons, muons and taus. The values obtained are compatible with each other, consistent with the principle of lepton universality. The mean leptonic branching ratio is found to be $0.0321 \pm$ 0.0013 , corresponding to a partial width $\Gamma_{\mathrm{a}}=85.4 \pm 5.3 \mathrm{MeV}$. The branching ratio and partial width for hadronic decays are found to be $0.689 \pm 0.030$ and $1833 \pm 116 \mathrm{MeV}$, respectively. The number of light neutrino species is deduced to be $3.35 \pm 0.41$ and the partial width for the invisible decay modes of the $\mathrm{Z}$ is found to be $569 \pm 92 \mathrm{MeV}$.

\section{Acknowledgement}

We wish to congratulate and thank our colleagues in the LEP division for their notable achievement in commissioning and operating the LEP accelerator. We wish to thank also the engineers and technicians in all our institutions for their enthusiastic and capable efforts which were vital for the construction of ALEPH. Those of us from non-member countries thank CERN for its hospitality. 


\section{References}

[1] LEP Design Report, Vol. I CERN-LEP/TH/83-9, and Vol. II CERN-LEP/TH/84-01.

[2] UA1 G. Arnison et al., Phys. Lett. 126 B (1983) 398;

UA2 P. Bagnaia et al., Phys. Lett. 129 B (1983) 130.

[3] ALEPH, D. Decamp et al., CERN EP/89-132 (1989).

[4] "ALEPH - A detector for electron-positron annihilation at LEP" to be published in Nucl.Inst. and Methods.

[5] M. Böhm, A. Denner and W. Hollik, Nucl. Phys. B304 (1988) 687;

F.A. Berends, R. Kleiss and W. Hollik, Nucl. Phys. B304 (1988) 712;

see also Monte Carlo Group Report, Z Physics at LEP, Vol. III, CERN 89-08, (1989).

[6] S. Jadach and Z. Was, Comp. Phys. Commun. 36 (1985) 191; see also Monte Carlo Group Report, Z Physics at LEP, Vol. III, CERN 89-08 (1989).

[7] Z Line Shape Group Report, Z Physics at LEP Vol. I CERN 89-08 (1989).

A. Blondel, CERN-EP 89-84 (1989).

[8] G. Feldman, Proceedings of the Third Mark II Workshop on SLC Physics, SLAC-315 (1987). 


\section{TABLE 1}

\section{Decay}

$$
\mathrm{Z} \rightarrow \mathrm{e}^{+} \mathrm{e}^{-}
$$$$
0.0472 \pm 0.0061
$$$$
\mathrm{Z} \rightarrow \mu^{+} \mu^{-}
$$$$
0.0435 \pm 0.0060
$$$$
0.0483 \pm 0.0051
$$$$
0.0466 \pm 0.0034
$$$$
\mathrm{Z} \rightarrow \mathrm{1}^{+} \mathrm{1}^{-}
$$

$\mathrm{Z} \rightarrow$ hadrons

$Z \rightarrow$ invisible
B

$\Gamma(\mathrm{MeV})$

$$
\begin{array}{ll}
0.0323 \pm 0.0021 & 85.9 \pm 6.9 \\
0.0298 \pm 0.0046 & 79.3 \pm 12.8 \\
0.0331 \pm 0.0041 & 88.0 \pm 11.7 \\
0.0321 \pm 0.0013 & 85.4 \pm 5.3 \\
0.689 \pm 0.030 & 1833 \pm 116 \\
0.214 \pm 0.029 & 569 \pm 92
\end{array}
$$

\section{Figure Captions}

1. A section through the ALEPH apparatus transverse to the beam direction, showing a $\tau$ pair decaying to a muon and three pions. The curvature of the muon in HCAL is opposite to that in the TPC since the magnetic field is in the opposite sense in HCAL which acts as the return path.

2. The angular distribution for $\mathrm{e}^{+} \mathrm{e}^{-} \rightarrow \mathrm{e}^{+} \mathrm{e}^{-}$showing the $\mathrm{t}$-channel peak in the forward direction.

3. Distance of closest approach to the beam line, $d$, versus distance along the beam line, $z$.

4. The distribution of distance along the beam, $z$ a) the preselected lepton sample

b) the final selected muon pairs.

5. The total charged energy distribution for the final $\tau^{+} \tau^{-}$sample. 


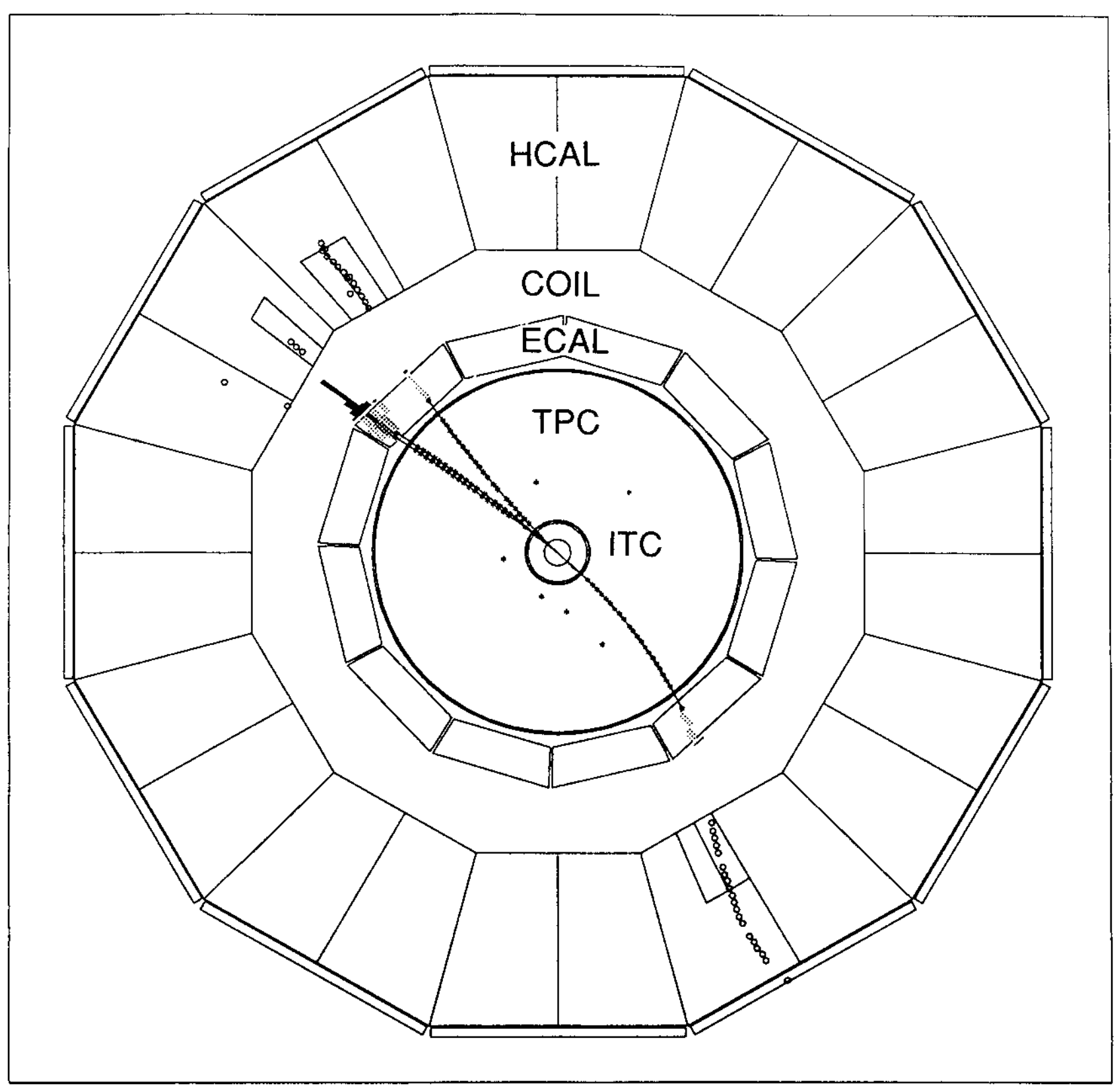

Figure 1 


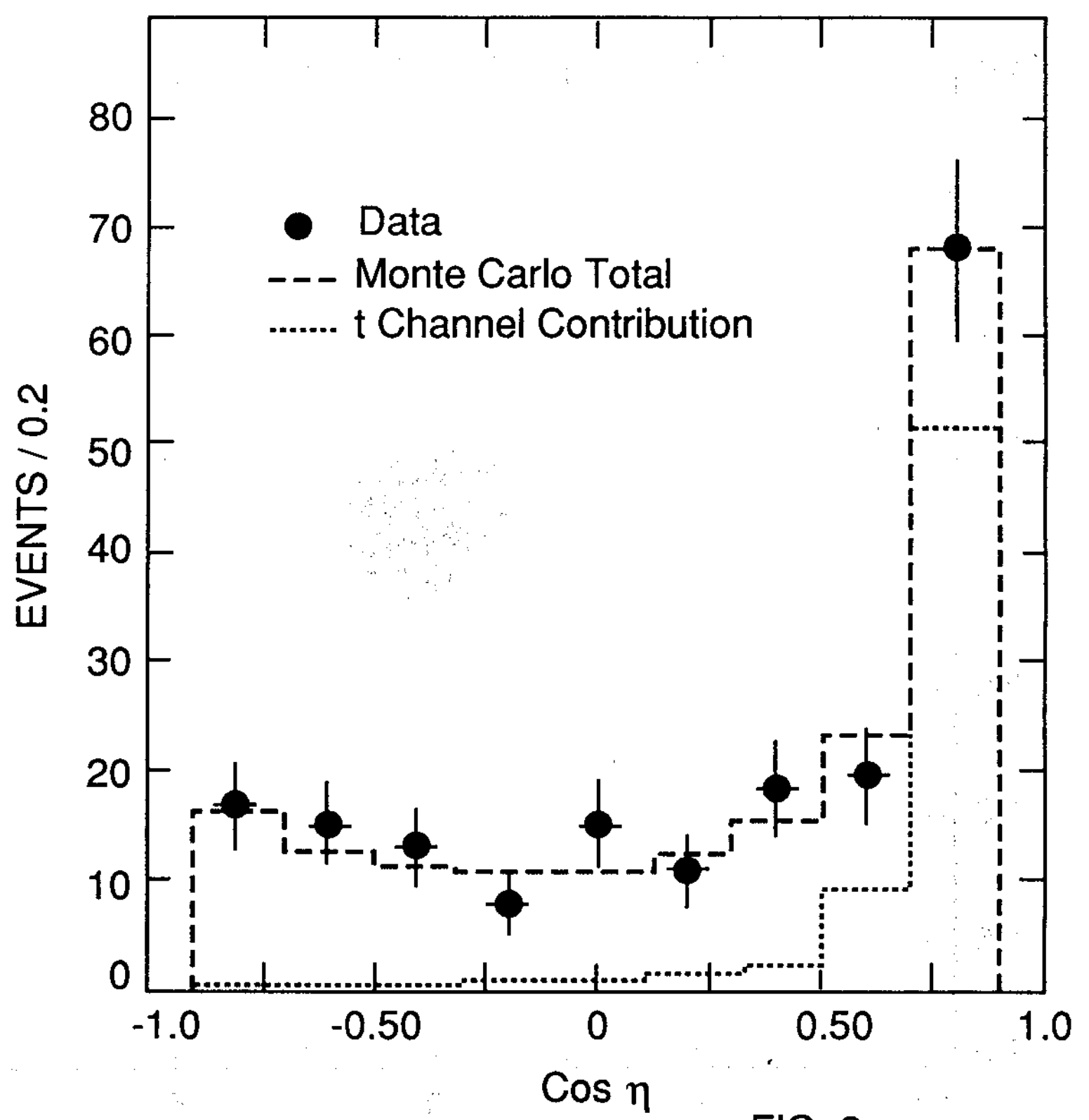

FIG. 2 


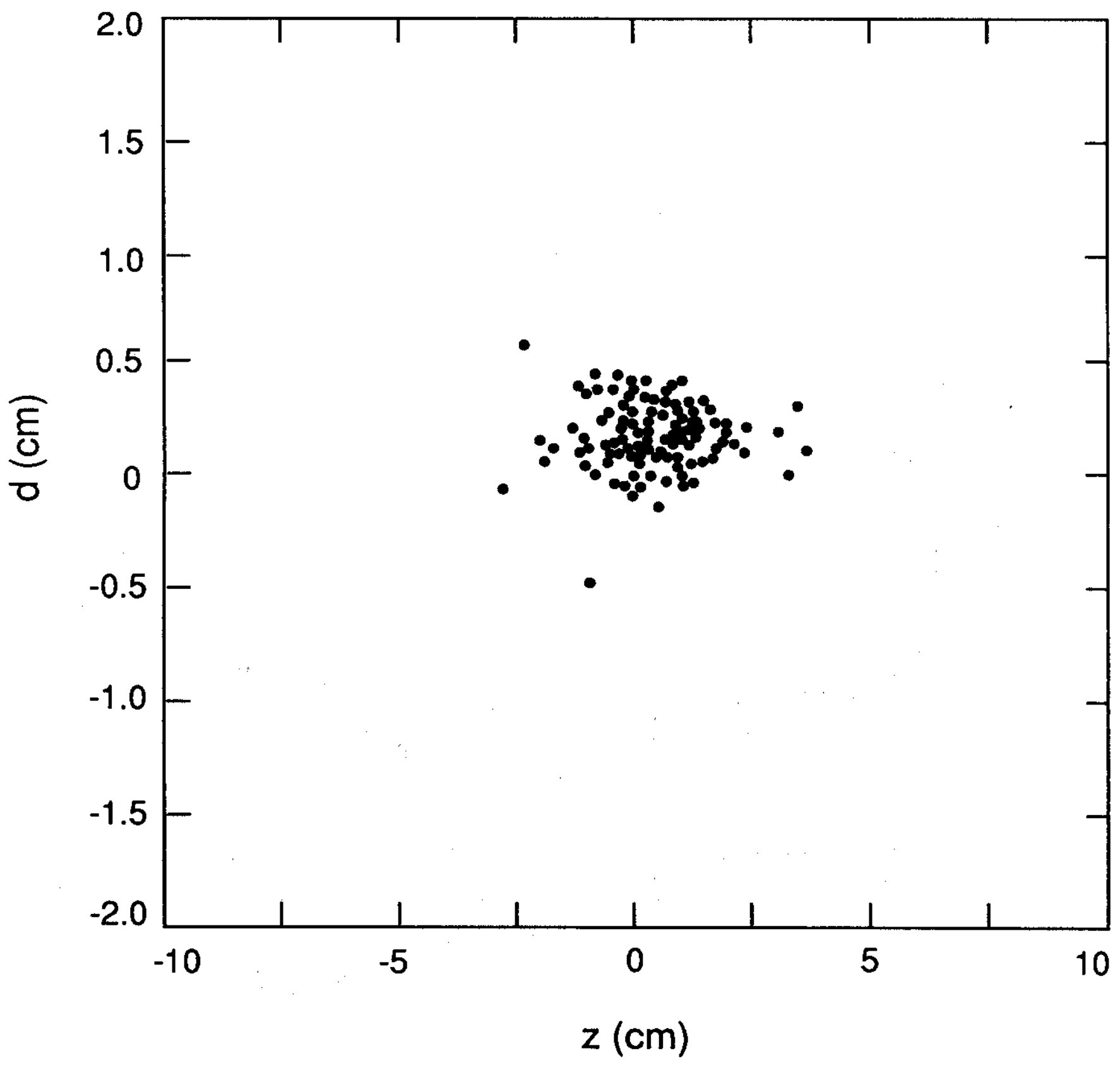

FIG. 3 

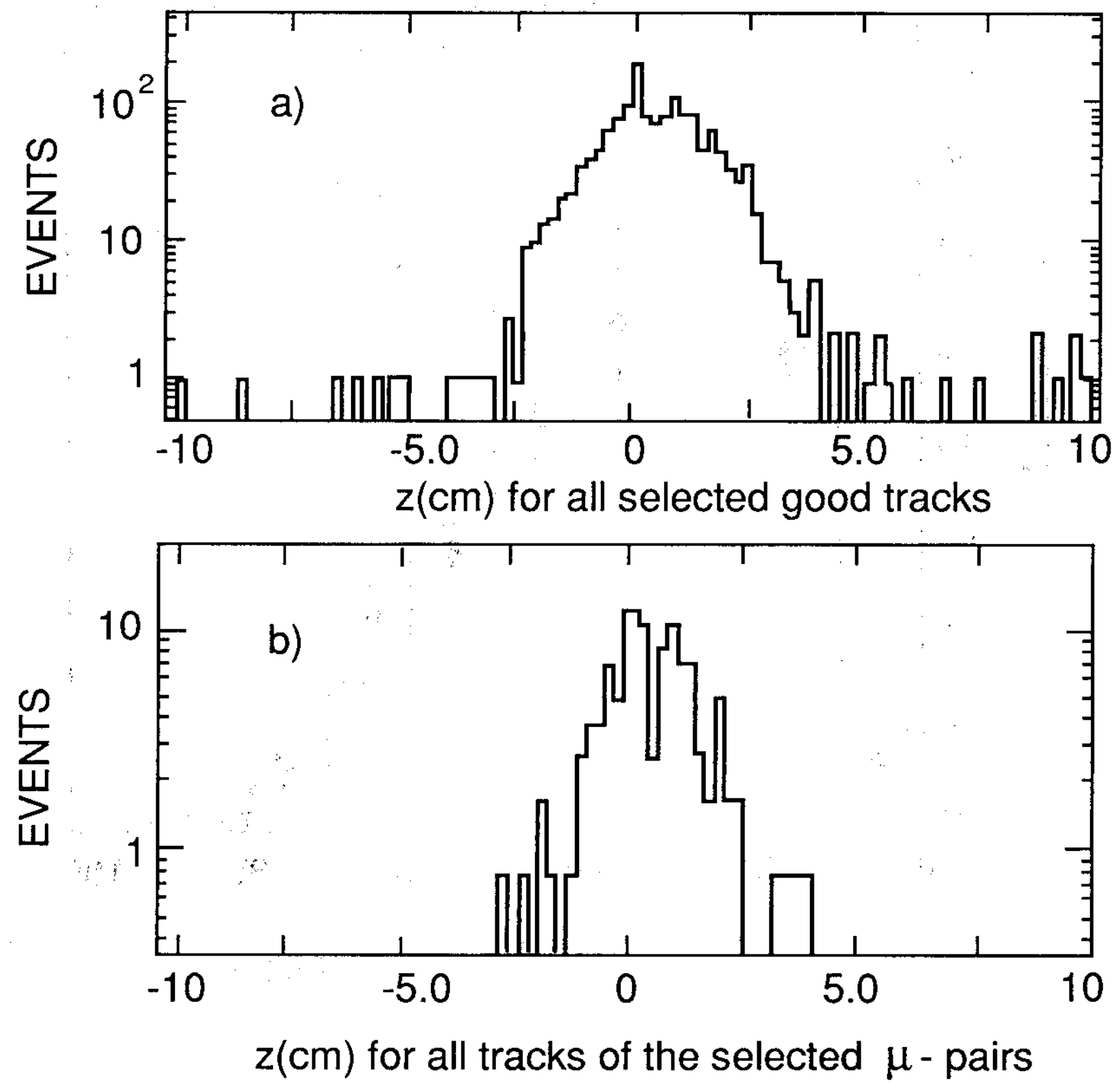

FIG. 4 


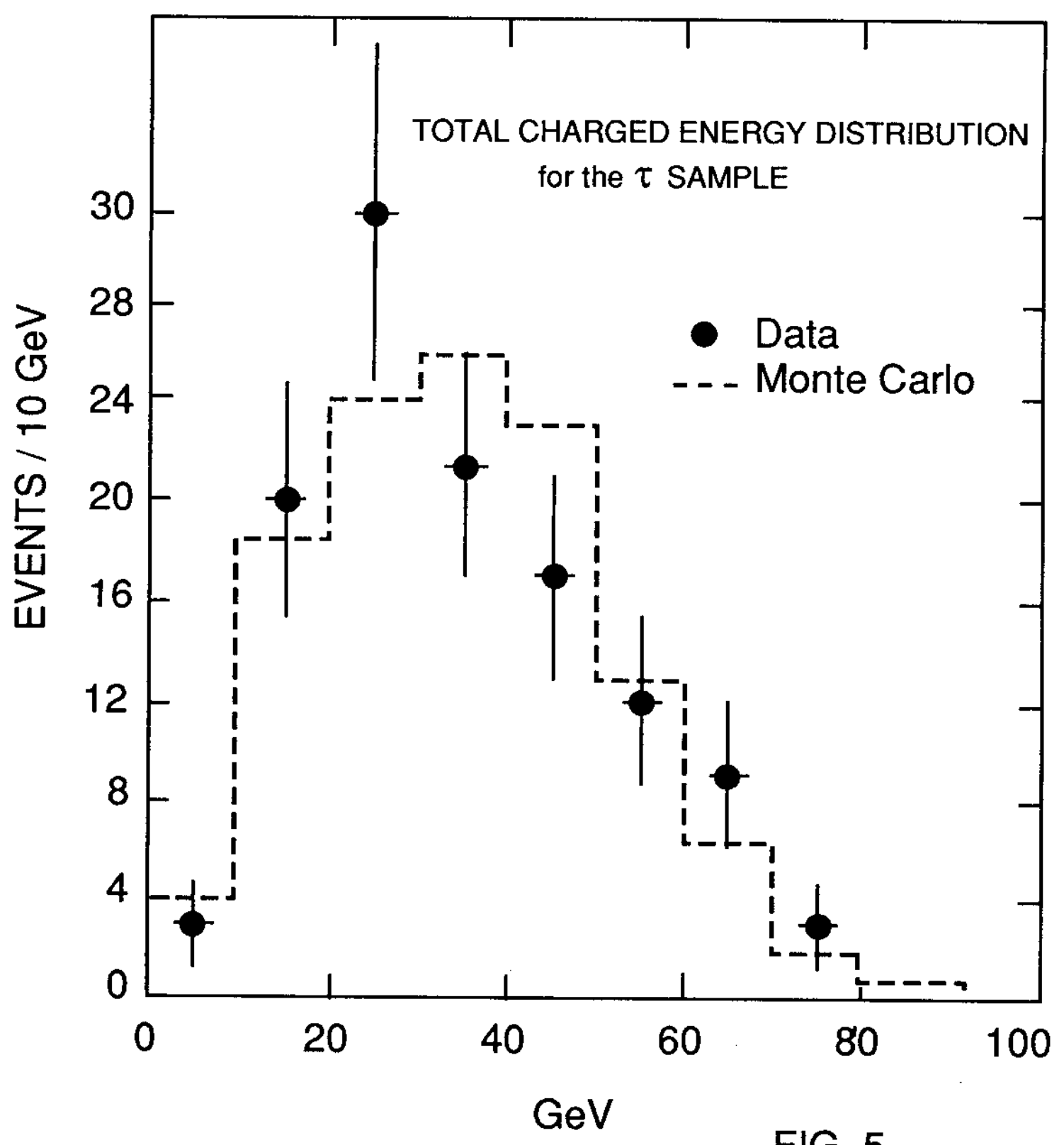

FIG. 5 